\title{
MicroRNA-1288 promotes cell proliferation of human glioblastoma cells by repressing ubiquitin carboxyl-terminal hydrolase CYLD expression
}

\author{
JUN YIN $^{1 *}$, CHENGYIN WENG $^{2 *}$, JIEKE MA $^{3}$, FANFAN CHEN $^{4}$, YECAI HUANG $^{1}$ and MEI FENG ${ }^{1}$ \\ ${ }^{1}$ Department of Radiation Oncology, Sichuan Cancer Hospital and Institute, Sichuan Cancer Center, School of Medicine, \\ University of Electronic Science and Technology of China, Chengdu, Sichuan $610041 ;{ }^{2}$ Department of Oncology, \\ Guangzhou First People's Hospital, Guangzhou Medical University, Guangzhou, Guangdong 510180; ${ }^{3}$ Department of \\ Craniofacial and Neurosurgery, Sichuan Cancer Hospital, Chengdu, Sichuan 610041; ${ }^{4}$ Department of Neurosurgery, \\ Guangzhou First People's Hospital, Guangzhou Medical University, Guangzhou, Guangdong 510180, P.R. China
}

Received October 13, 2016; Accepted June 21, 2017

DOI: $10.3892 / \mathrm{mmr} .2017 .7481$

\begin{abstract}
Previous studies have demonstrated that microRNAs (miRs) are important regulators involved in various cancers, including human glioblastoma (GBM). However, the underlying mechanism of miR-1288 remains poorly understood, and its role in GBM has not been reported. The present study confirmed that miR-1288 expression was markedly upregulated in GBM. Ectopic expression of miR-1288 promoted the proliferation, colony formation and anchorage-independent growth of GBM cells. Bioinformatics analysis coupled with western blotting and luciferase report assays also indicated that miR-1288 promoted cell proliferation of GBM by targeting ubiquitin carboxyl-terminal hydrolase (CYLD). Knockdown of CYLD expression reversed the cell proliferation promotion by miR-1288-in in GBM. These results suggest that the miR-1288/CYLD axis may represent a potential therapeutic target for the treatment of GBM.
\end{abstract}

\section{Introduction}

Human glioblastoma (GBM) is the most frequent and aggressive type of primary brain tumor in adults, and remains an important health problem worldwide (1). Although advances in the treatment strategies of GBM have been made, the 5-year survival rate of GBM patients remains poor (2). Thus, it is

Correspondence to: Dr Jun Yin, Department of Radiation Oncology, Sichuan Cancer Hospital and Institute, Sichuan Cancer Center, School of Medicine, University of Electronic Science and Technology of China, 55 Renmin South Road, Chengdu, Sichuan 610041, P.R. China

E-mail: scchyinjun@163.com

*Contributed equally

Key words: microRNA-1288, glioblastoma, ubiquitin carboxyl-terminal hydrolase CYLD, cell proliferation important to investigate novel therapeutic strategies for GBM treatment.

Accumulating studies have demonstrated that microRNAs (miRNAs/miRs), a class of small and non-coding RNAs, participate in various biological processes of cancer development by binding to the 3'-untranslated region (3'-UTR) of mRNA (3-5). For example, miR-300 was reported to serve as a tumor suppressor gene and suppress GBM progression by ROCK1 (6). miR-595 was demonstrated to serve a critical role in GBM carcinogenesis by suppression of transcription factor SOX7 (7). Chen et al (8) indicated that miR-22 suppresses cell proliferation, motility and invasion of GBM by directly targeting NAD-dependent protein deacetylase sirtuin-1. To date, the role of miR-1288 in GBM remains unclear. Gopalan et al (9) revealed that overexpression of miR-1288 in colon cancer cells promotes cell proliferation and increases the percentage of G2-M phase cells. In addition, they demonstrated that miR-1288 overexpression increases cell proliferation and colony formation, and enhances cell migration and cell invasion properties in oesophageal squamous cell carcinoma by regulating forkhead box protein O1 (10). The present study observed aberrantly increased expression of miR-1288 in GBM tissues/cells. Next, we experimentally revealed that miR-1288 acted as a tumor promoter by interacting with ubiquitin carboxyl-terminal hydrolase CYLD (CYLD), and then regulating cell proliferation in GBM.

\section{Materials and methods}

Clinical specimens. Eight paired surgically-removed human glioblastoma tissues were obtained from GBM patients, and two normal brain tissues were obtained from individuals who passed away in traffic accidents, and then histopathologically diagnosed at Sichuan Cancer Hospital (Sichuan, China). The present study was approved by the ethics committee of Sichuan Cancer Hospital. All samples were collected and analyzed with prior written informed consent from the patients. Tissue samples were frozen in liquid nitrogen and stored until total RNAs or proteins were extracted. 
miR-1288 expression profiles (GSE61710) in GBM tissues were obtained from Gene Expression Omnibus (GEO; www. ncbi.nlm.nih.gov/geo/). Archived patient samples in The Cancer Genome Atlas (TCGA) database (https://tcga-data.nci. nih.gov and https://genomecancer.ucsc.edu) were selected.

Cell culture. The LN229, LN18, U87MG, A172, D27MG and LN340 human GBM cell lines were provided by the National Rodent Laboratory Animal Resource (Shanghai, China) and were grown in Dulbecco's modified Eagle's medium (Gibco; Thermo Fisher Scientific, Inc., Waltham, MA, USA) supplemented with $10 \%$ fetal bovine serum (FBS; Sigma-Aldrich; Merck KGaA, Darmstadt, Germany). Normal human astrocyte (NHA) cells were obtained from Lonza Group, Ltd. (Basel, Switzerland) and cultured in the provided astrocyte growth media supplemented with recombinant human epidermal growth factor, insulin, ascorbic acid, GA-1000, L-glutamine and $5 \%$ FBS. Cell lines were cultured in a humidified incubator at $37^{\circ} \mathrm{C}$ in $5 \% \mathrm{CO}_{2}$.

RNA extraction and reverse transcription-quantitative polymerase chain reaction ( $R T-q P C R)$. Total RNA including miRNAs was extracted from human tissue samples and cell lines using TRIzol reagent (Thermo Fisher Scientific, Inc.). RNA was reverse transcribed to cDNA from RNA using a Reverse Transcription kit (Takara Biotechnology Co., Ltd., Dalian, China) according to the manufacturer's protocol.qPCR was performed with SYBR Green (Takara Biotechnology Co., Ltd.) on a ABI 7500 thermocycler. Thermocycling conditions were as follows: At $95^{\circ} \mathrm{C}$ for $30 \mathrm{sec}$, followed by 40 cycles of amplification at $95^{\circ} \mathrm{C}$ for $5 \mathrm{sec}$, at $59^{\circ} \mathrm{C}$ for $30 \mathrm{sec}$ and at $72^{\circ} \mathrm{C}$ for $30 \mathrm{sec}$. The sequences of primers were synthesized by GeneCopoeia, Inc. (Rockville, MD, USA): miR-1288 (cat. no. HmiRQP0132), cyclin D1 (cat. no. HQP016204) and MYC (cat. no. HQP011597). U6 and GAPDH (cat. no. HQP064347) were used as endogenous controls for miRNA and mRNA, respectively, and the data were analyzed according to the $2^{-\Delta \Delta \mathrm{Cq}}$ method (11).

Plasmids, small interfering RNA and transfection. miR-1288 mimics (miR10005942-1-5), a miR-1288 inhibitor (miR-1288-in; miR20005942-1-5), negative control sequences and CYLD-specific small interfering (si)RNA and scramble sequences were synthesized and purified by Guangzhou RiboBio Co., Ltd. (Guangzhou, China), and transfection into cells was performed using Lipofectamine 2000 (Invitrogen; Thermo Fisher Scientific, Inc.) according to the manufacturer's protocol.

MTT and colony formation assays. Cell growth was measured by MTT assay. Cells were seeded at $5 \times 10^{4}$ cells/well into a 24-well plate after each transfection. After 1, 2, 3, 4 and 5 days of culture at $37^{\circ} \mathrm{C}$, cell viability was assessed by MTT assay. MTT solution ( $20 \mu \mathrm{l} ; 0.5 \mathrm{mg} / \mathrm{ml}$; Sigma-Aldrich; Merck $\mathrm{KGaA}$ ) was added to each well at $37^{\circ} \mathrm{C}$. The medium was removed from each well and the formazan was solubilized in $150 \mu$ l dimethyl sulfoxide (Sigma-Aldrich; Merck KGaA). The absorbance of each well at $490 \mathrm{~nm}$ was measured using a Multiskan GO microplate spectrophotometer (Thermo Fisher Scientific, Inc.).
For the colony formation assay, a density of 1,000 indicated U87MG cells/well upon different treatments were seeded into a 6-well cell culture plate and incubated for 14 days at room temperature. Colonies were fixed with $4 \%$ paraformaldehyde for $5 \mathrm{~min}$ and stained at room temperature with $1.0 \%$ crystal violet for $30 \mathrm{sec}$. The number of colonies was counted under a microscope (Motic AE30 inverted fluorescence microscope; Microscope Systems Limited, Glasgow, UK) at magnification, $\mathrm{x} 100$.

Anchorage-independent growth assay. Cells (1,000) were suspended in $2 \mathrm{ml}$ complete medium plus $0.3 \%$ agar (Sigma-Aldrich; Merck KGaA) and then plated on top of a bottom layer containing $0.5 \%$ complete medium agar mixture. After 14 days, viable colonies that were larger than $0.1 \mathrm{~mm}$ in diameter were counted by microscopy (Motic AE30 inverted fluorescence microscope; Microscope Systems Limited).

Bromodeoxyuridine (BrdU) staining. U87MG cells after transfection were incubated with $10 \mu \mathrm{M}$ BrdU for $1 \mathrm{~h}$ and for an extra hour after the transfer to fresh medium, fixed with $4 \%$ paraformaldehyde for $30 \mathrm{~min}$ at room temperature and then stained at $4^{\circ} \mathrm{C}$ overnight with BrdU antibodies (1:500; cat no. 61273; Upstate Biotechnology, Inc., Lake Placid, NY, USA) according to the manufacturer's protocol. After incubation at $37^{\circ} \mathrm{C}$ for $1 \mathrm{~h}$ with horseradish peroxidase (HRP)-conjugated secondary antibodies (1:5,000; Abcam, Cambridge, UK), images were acquired using a laser scanning microscope (Axioskop 2 plus; Zeiss GmbH, Jena, Germany). All experiments were performed at least three times.

MiRNA target prediction and luciferase assays. Potential target genes of miR-1288 were predicted using TargetScan online software version 3.1 (www.targetscan.org). Cells were co-transfected with the wild-type CYLD3'UTR (GeneCopoeia, Inc.) or the control-luciferase plasmid and miR-1288 or miR-1288-mutant (mut). Each group was also co-transfected with $5 \mathrm{ng}$ control pRL-TK Renilla plasmid (Promega Corporation, Madison, WI, USA) using Lipofectamine 2000 (Invitrogen; Thermo Fisher Scientific, Inc.). Luciferase and Renilla activities were assessed $48 \mathrm{~h}$ after transfection using a Dual Luciferase Reporter Assay kit (Promega Corporation) according to the manufacturer's protocol.

Western blot analysis. Protein lysates were prepared using radioimmunoprecipitation assay lysis buffer (Cell Signaling Technology, Inc., Danvers, MA, USA). The protein concentration was determined using a bicinchoninic acid protein assay. Equal amounts $(40 \mu \mathrm{g})$ of proteins were separated by $10 \%$ SDS-PAGE and electrotransferred onto polyvinylidene difluoride membranes (EMD Millipore, Billerica, MA, USA). Membranes were subsequently blocked in TBS containing $0.5 \%$ Tween-20 with $5 \%$ milk for $2 \mathrm{~h}$ at room temperature and incubated at $4{ }^{\circ} \mathrm{C}$ with the following primary antibodies overnight: Anti-CYLD (cat no. 8462; 1:1,000), anti-cyclin D1 (cat no. 2978; 1:1,000) and anti-c-MYC (cat no. 5605; 1:1,000; all Cell Signaling Technology, Inc.). An anti- $\alpha$-tubulin monoclonal antibody (cat no. T6199; 1:5,000; Sigma-Aldrich; Merck KGaA) served as a loading control. The blots were then incubated at $37^{\circ} \mathrm{C}$ for $2 \mathrm{~h}$ with 

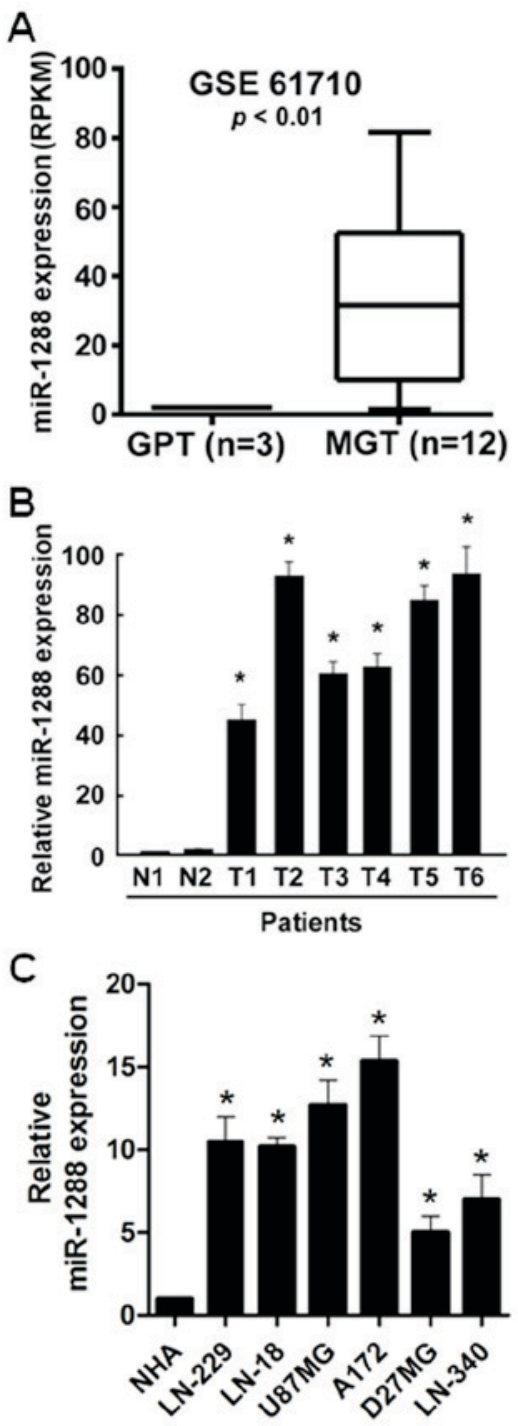

Figure 1. Expression of miR-1288 in human GBM tissues and cell lines. (A) The expression levels of miR-1288 in GBM tissues from the Gene Expression Omnibus (GSE61710). Reverse transcription-quantitative polymerase chain reaction analysis of miR-1288 expression levels in (B) 6 primary GBM tissues and normal brain tissues. ${ }^{*} \mathrm{P}<0.05$ vs. $\mathrm{N} 1$ or N2. (C) miR-1288 expression levels in NHA and GBM cell lines. Data are presented as the mean \pm standard deviation of three independent experiments. ${ }^{*} \mathrm{P}<0.05$ vs. NHA. T, primary GBM tissues; $\mathrm{N}$, normal brain tissues; NHA, normal human astrocyte; GBM, glioblastoma; miR, microRNA; MGT, malignant glioma tissues; GPT, glioma peritumoral tissues; GSE, Gene Expression Omnibus accession number; RPKM, reads per kilobase per million reads.

a HRP-conjugated anti-rabbit immunoglobulin secondary antibody (cat no. P0023D; 1:5,000; Beyotime Institute of Biotechnology, Haimen, China). The signals were visualized using enhanced chemiluminescence following the manufacturer's protocol.

Statistical analysis. All data are expressed as the mean \pm standard deviation. The data were analyzed using Student's t-test for pair-wise comparisons or one-way analysis of variance followed by a post hoc Tukey test for multiple comparisons. Statistical analysis was performed using SPSS 19.0 software (IBM Corp., Armonk, NY, USA). P<0.05 was considered to indicate a statistically significant difference.
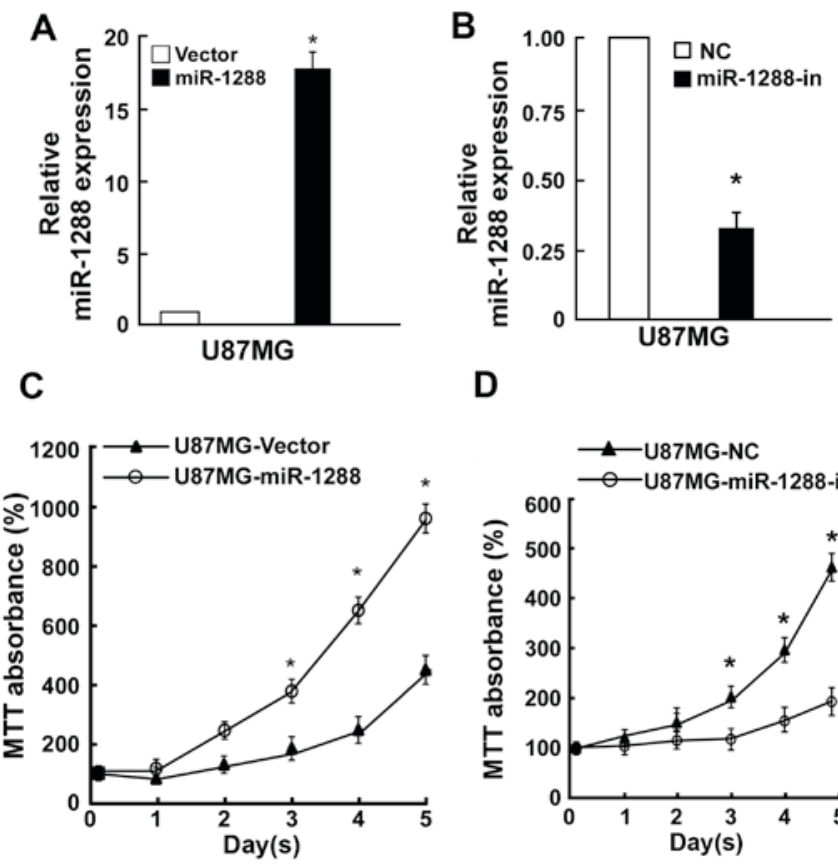

D

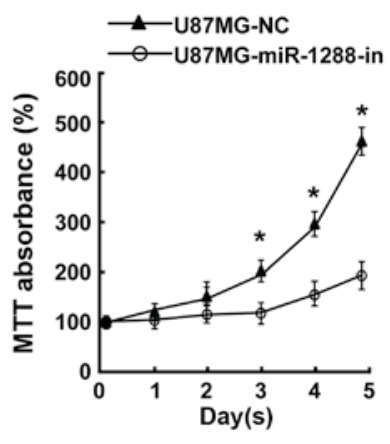

Figure 2. miR-1288 transfection effects on U87MG human glioblastoma cells. Validation of miR-1288 expression levels after transfection with (A) vector and miR-1288, and (B) NC and miR-1288-in by reverse transcription-quantitative polymerase chain reaction analysis. ${ }^{*} \mathrm{P}<0.05$ vs. vector. MTT assays of U87MG cells transfected with (C) vector and miR-1288, and (D) NC and miR-1288-in. " $\mathrm{P}<0.05$ vs. vector group. Data are presented as the mean \pm standard deviation of three independent experiments. miR, microRNA; miR-1288-in, microRNA-1288-inhibitor; NC, negative control.

\section{Results}

miR-1288 expression is upregulated in GBM. To reveal the role of miR-1288 in GBM, the expression data downloaded from the Gene Expression Omnibus (GEO, accession number GSE61710) was analyzed. The results of the GEO analysis demonstrated that miR-1288 levels were significantly increased in malignant glioma tissues compared with glioma peritumoral tissues (Fig. 1A). To further confirm this observation, the expression of miR-1288 in human glioblastoma tissues and normal brain tissues was measured by RT-qPCR. miR-1288 expression was significantly upregulated in GBM tissues compared with normal brain tissues (Fig. 1B). The expression of miR-1288 in LN229, LN443, LN18, U87MG, A172, D27MG and LN340 GBM cells was further detected, and the result indicated that compared with NHA cells, miR-1288 expression was significantly increased in GBM cells (Fig. 1C). miR-1288 expression in U87MG cells was less abundant than in A172 cells, but was more abundant compared with the other GBM cell lines. Therefore, the U87MG cell line may the most appropriate model for studying the expression of miR-1288 in relation to GBM.

miR-1288 promotes, while miR-1288-in inhibits GBM cell proliferation. To investigate whether GBM cell proliferation was regulated by miR-1288, U87MG cells were cotransfected with miR-1288 or miR-1288-in or the respective controls. RT-qPCR analysis was used to verify relative miR-1288 expression (Fig. 2A and B). MTT assay revealed that ectopic overexpression of miR-1288 markedly enhanced cell proliferation 
A
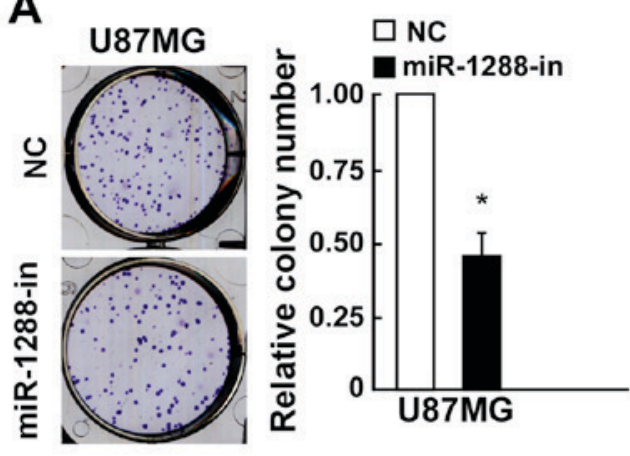

C
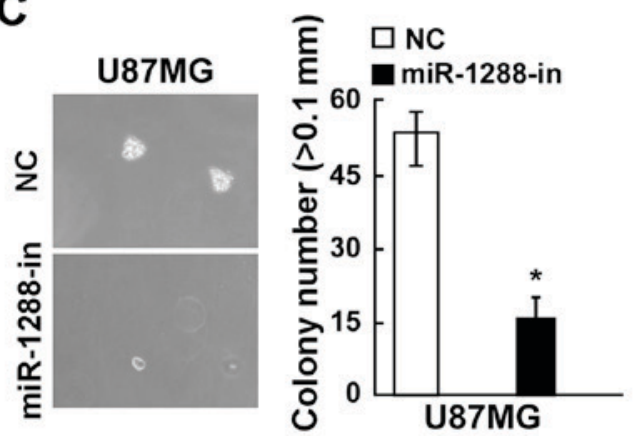

B

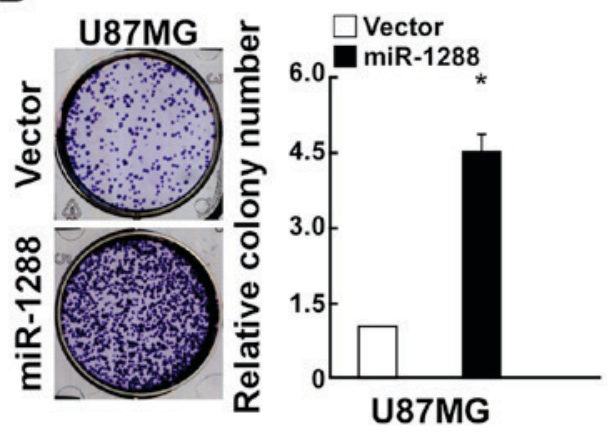

D U87MG
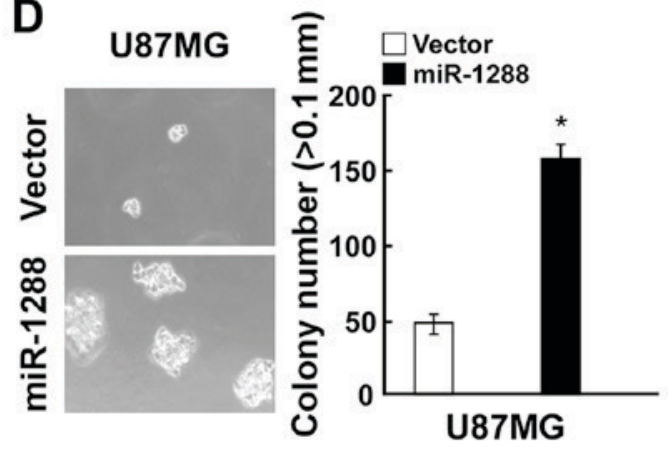

E
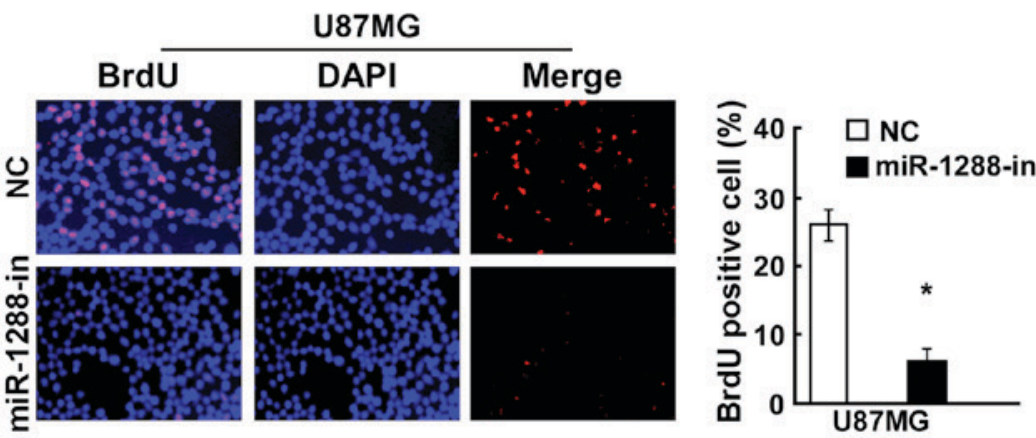

$\mathbf{F}$
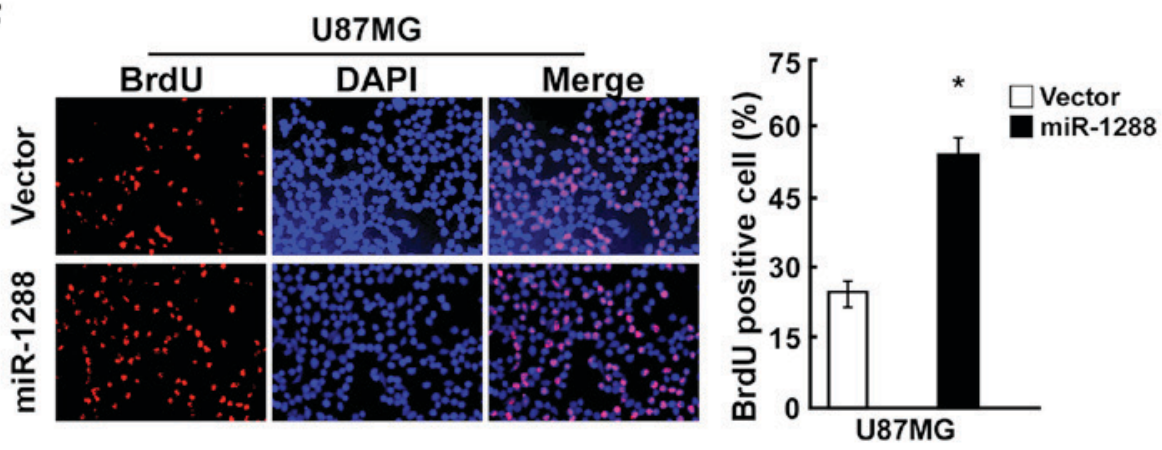

Figure 3. miR-1288 transfection effects on U87MG human glioblastoma cells. Representative micrographs and quantification of crystal violet-stained cells transfected with (A) NC and miR-1288-in, and (B) vector and miR-1288. Colony formation of cells transfected with (C) NC and miR-1288-in and (D) vector and miR-1288. Magnification, x100. Representative micrographs and quantification of the BrdU incorporation assay in cells transfected with (E) NC and miR-1288-in, and (F) vector and miR-1288. Magnification, x100. Data are presented as the mean \pm standard deviation. "P $<0.05$. BrdU, bromodeoxyuridine; miR, microRNA; miR-1288-in, microRNA-1288-inhibitor; NC, negative control.

of U87MG cells, while suppression of cell proliferation by miR-1288-in (Fig. 2B and C). Similarly, a colony formation assay demonstrated that compared with the relative negative control, miR-1288 significantly promoted while miR-1288-in markedly suppressed colony formation of U87MG cells (Fig. 3A and B). Additionally, the anchorage-independent growth ability of U87MG cells transfected with miR-1288 was significantly increased, and miR-1288-in treatment caused a decrease in the anchorage-independent growth ability of U87MG cells (Fig. 3C and D). BrdU assay results demonstrated that BrdU-positive cells were significantly increased in the U87MG cell line after transfection with miR-1288, while miR-1288-in exhibited the opposite effect (Figs. 2E and 3E). These results demonstrated that miR-1288 increases GBM cell tumorigenicity in vitro. 
A CYLD-3'UTR $5^{5} 799^{\circ}$ UCCUUUACAAACAUACAGUCCAU ${ }^{8123}$ IIIIIII

miR-1288 3.AGAGGUCUAGUCCCGUCAGGU 5 miR-1288 mut 3.AGAGGUCUAGUCCCGUCCAUU $5^{\circ}$

B

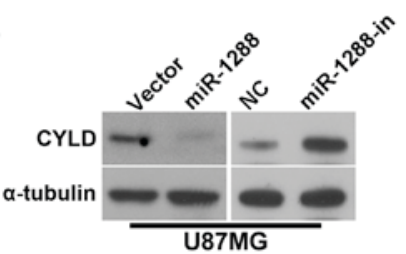

C

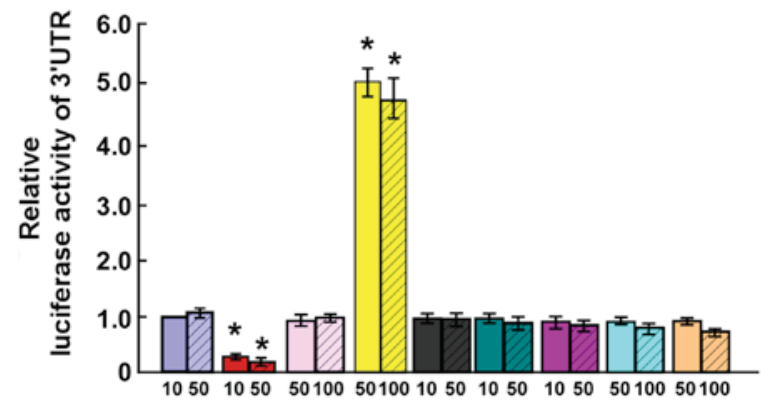

NC (for miR-1288 inhibitor) - - - + + - - - - - + + -

miR-1288-in _ - - - + + . - - - +

Vector $++--_{-}--_{-}+--_{-}-{ }_{-}$

miR-1288 - - + + - - - - - - + + - - -

miR-1288-mut - - - - - - - + + - - - - - +

pGL3-CYLD-3'UTR $+++++++++-\cdots--$ - - -

U87MG

\section{D}

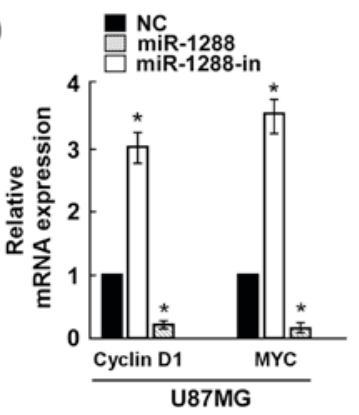

E

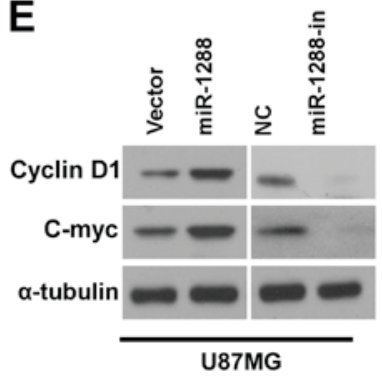

Figure 4. miR-1288 suppresses CYLD expression by directly targeting the CYLD 3'-UTR and altered levels of proteins associated with proliferation in U87MG cells. (A) Predicted miR-1288 target sequence in the 3'-UTR of CYLD and positions of three mutated nucleotides (red) in the miR-1288-mut. (B) CYLD protein expression levels in U87MG cells transfected with miR-1288 or the miR-1288 inhibitor were detected by western blotting analysis. $\alpha$-tubulin served as the loading control. (C) Luciferase reporter assay of U87MG cells transfected with the pGL3-CYLD-3'-UTR reporter and miR-1288 or miR-1288-in. (D) mRNA and (E) protein expression levels of cyclin D1 and MYC, as assessed by reverse transcription-quantitative polymerase chain reaction and western blot analyses. Data are presented as the mean \pm standard deviation. ${ }^{*} \mathrm{P}<0.05$. mut, mutant; miR, microRNA; miR-1288-in; microRNA-1288-inhibitor; NC, negative control; UTR untranslated region; CYLD, ubiquitin carboxyl-terminal hydrolase CYLD.

miR-1288 directly targets CYLD by binding to its 3'-UTR. Bioinformatics methods (www.targetscan.org) identified CYLD as a potential target of miR-1288 (Fig. 4A). To elucidate whether CYLD is indeed directly targeted by miR-1288, the present study investigated whether miR-1288 recognizes the 3'UTR of CYLD mRNA using western blot analysis and dual-luciferase reporter assay. Western blot analysis demonstrated that CYLD was decreased in miR-1288-transfected U87MG cells, while miR-1288-in clearly increased CYLD

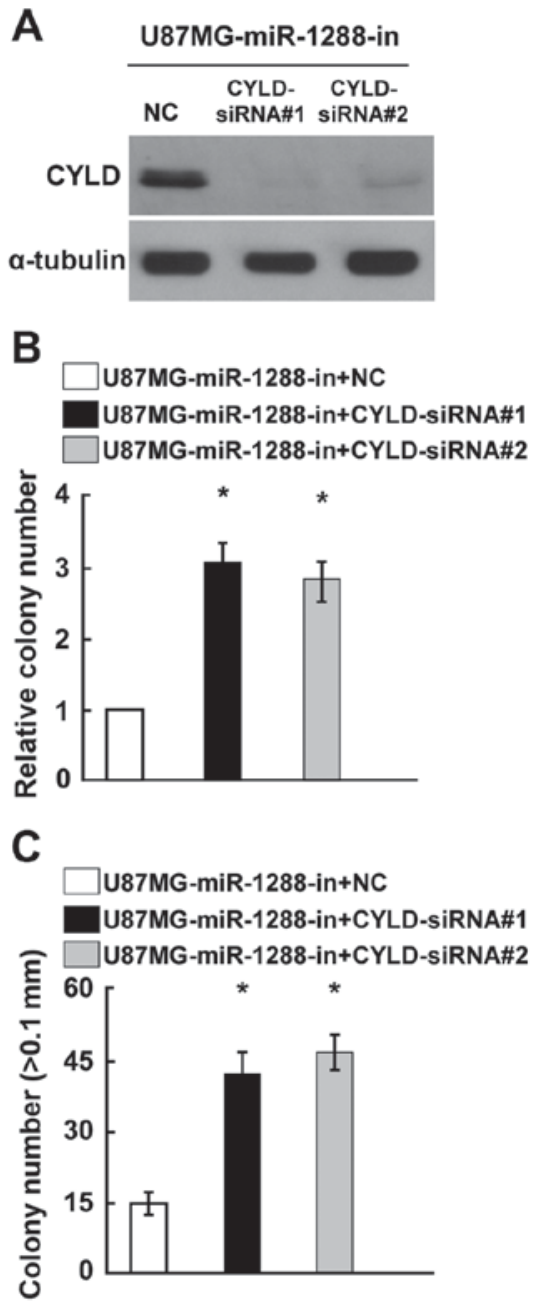

Figure 5. Silencing of CYLD expression reverses the cell proliferation promoted by miR-1288-in in glioblastoma. (A) Western blot analysis verified that silencing CYLD effectively decreased the expression of CYLD in miR-1288-in-transfected U87MG cells. (B) miR-1288-in-transfected U87MG cells increased in cell colony formation after transfection with CYLD-siRNAs. (C) Representative quantification of colonies that were $>0.1 \mathrm{~mm}$. Data are presented as the mean \pm standard deviation of three independent experiments. ${ }^{*} \mathrm{P}<0.05$ vs. miR-1288-in+NC. miR, microRNA; miR-1288-in, microRNA-1288-inhibitor; NC, negative control; siRNA, small interfering RNA; CYLD, ubiquitin carboxyl-terminal hydrolase CYLD.

protein expression (Fig. 4B). A CYLD 3'-UTR wild-type vector was co-transfected into U87MG cells with miR-1288, miR-1288-in or miR-NC, and then measured by luciferase activity. The results revealed that transfection of miR-1288 markedly suppressed the luciferase activity of CYLD 3'-UTR wild-type in U87MG cells, transfection of miR-1288-in significantly increased the luciferase activity of CYLD 3'-UTR wild -type in U87MG cells, and miR-1288-mut demonstrated no effect on the luciferase activity of CYLD 3'-UTR wild-type in U87MG cells (Fig. 4C). Taken together, these results suggested that CYLD is a target of miR-1288.

To further determine the mechanism by which the miR-1288-CYLD axis regulates cell proliferation of GBM, the expression of cell proliferation-associated genes were measured. Decreased cyclin D1 and MYC expression were detected after treatment with the miR-1288 by RT-qPCR and western blot analysis (Fig. 4D and E, respectively). Collectively, 
these results indicated that miR-1288 functionally modulates the cellular proliferation regulators cyclin D1 and MYC; thus, may mediate GBM cell proliferation.

Silencing of CYLD expression reversed the cell proliferation promotion by miR-1288-in in GBM. The present study hypothesized that the phenotypes associated with miR-1288-in expression would be reversed by the silencing of CYLD expression. CYLD expression was confirmed by western blotting (Fig. 5A). Colony formation and anchorage-independent growth assays demonstrated that knockout of CYLD expression significantly reversed the miR-1288-in-induced promotion of GBM cell proliferation (Fig. 5B and C). Taken together, these results indicated that CYLD serves an essential role in cell proliferation of GBM, potentially acting as a mediator of miR-1288.

\section{Discussion}

The results of the present study revealed the following novel findings: i) miR-1288 was upregulated in GBM; ii) in vitro experiments confirmed that miR-1288 overexpression promoted cell proliferation of GBM; iii) miR-1288 targeted CYLD in GBM cells and cells negatively expressing CYLD.

Previous studies have demonstrated that miRNAs serve essential roles in tumor development and progression of various types of cancer by targeting genes associated with cell proliferation, apoptosis, invasion, migration and angiogenesis $(3,12-16)$. miR-146b-5p serves as a tumor suppressor and predicts the prognosis of human gliomas (17). miR-182-5p was reported to promote tumorigenesis of glioma by inducing STAT3 activation (18). Guo et al (19) indicated that miR-141 and miR-200c inhibit glioma cell growth and migration by suppressing zinc finger E-box-binding homeobox 1 expression. In particular, the function of $\mathrm{miR}-1288$ and its regulated targets in GBM remains unknown. The present study demonstrated that expression of miR-1288 was significantly upregulated in GBM tissues and cells.

miR-1288 has previously been reported to be associated with pathological staging and serve an essential role in the progression of colorectal cancer (9). Gopalan et al (20) demonstrated that miR-1288 expression was upregulated in oesophageal squamous cell carcinoma tissues, and overexpression of miR-1288 promoted cell proliferation, migration and invasion of oesophageal squamous cell carcinoma. Similarly, the present study indicated that overexpression of miR-1288 could promote cell proliferation of GBM in vitro.

CYLD, a mutated gene in familial cylindromatosis, is known to serve as tumor suppressor gene in multiple types of cancer by regulating various signaling pathways, including Wnt $/ \beta$-catenin, nuclear factor- $\kappa \mathrm{B}$ and transforming growth factor- $\beta$ (21-25). In the present study, CYLD was demonstrated to serve as a functional target of miR-1288, using a bioinformatics prediction. Western blotting and a dual-luciferase reporter assay were used to verify that miR-1288 targets CYLD by interacting with the 3'UTR of CYLD to reduce CYLD expression. Further functional experiments revealed that the suppression of CYLD reversed the cell proliferation promotion by miR-1288-in in GBM.

In conclusion, the present study demonstrated that miR-1288 expression was increased in GBM. miR-1288 was identified as a tumor promoter in GBM by inhibiting of CYLD, suggesting that the miR-1288/CYLD axis may represent a potential therapeutic target for the treatment of GBM.

\section{Acknowledgements}

The present study was supported by the Department of Radiation Oncology, Sichuan Cancer Hospital (Sichuan, China) and the Research project of Sichuan Provincial Health and Family Planning Commission (grant no. 16PJ511).

\section{References}

1. Van Meir EG, Hadjipanayis CG, Norden AD, Shu HK, Wen PY and Olson JJ: Exciting new advances in neuro-oncology: The avenue to a cure for malignant glioma. CA Cancer J Clin 60: 166-193, 2010.

2. Franceschi E, Depenni R, Paccapelo A, Ermani M, Faedi M, Sturiale C, Michiara M, Servadei F, Pavesi G, Urbini B, et al: Which elderly newly diagnosed glioblastoma patients can benefit from radiotherapy and temozolomide? A PERNO prospective study. J Neurooncol 128: 157-162, 2016.

3. Wang W, Chen J, Dai J, Zhang B, Wang F and Sun Y: MicroRNA-16-1 inhibits tumor cell proliferation and induces apoptosis in A549 non-small cell lung carcinoma cells. Oncol Res 24: 345-351, 2016.

4. Zhou W, Bi X, Gao G and Sun L: miRNA-133b and miRNA-135a induce apoptosis via the JAK2/STAT3 signaling pathway in human renal carcinoma cells. Biomed Pharmacother 84: 722-729, 2016.

5. Liu F, Zhang S, Zhao Z, Mao X, Huang J, Wu Z, Zheng L and Wang Q: MicroRNA-27b up-regulated by human papillomavirus 16 E7 promotes proliferation and suppresses apoptosis by targeting polo-like kinase 2 in cervical cancer. Oncotarget 7: 19666-19679, 2016.

6. Zhou F, Li Y, Hao Z, Liu X, Chen L, Cao Y, Liang Z, Yuan F, Liu J, Wang J, et al: MicroRNA-300 inhibited glioblastoma progression through ROCK1. Oncotarget 7: 36529-36538, 2016.

7. Hao Y, Zhang S, Sun S, Zhu J and Xiao Y: MiR-595 targeting regulation of SOX7 expression promoted cell proliferation of human glioblastoma. Biomed Pharmacother 80: 121-126, 2016.

8. Chen H, Lu Q, Fei X, Shen L, Jiang D and Dai D: miR-22 inhibits the proliferation, motility, and invasion of human glioblastoma cells by directly targeting SIRT1. Tumour Biol 37: 6761-6768, 2016.

9. Gopalan V, Pillai S, Ebrahimi F, Salajegheh A, Lam TC, Le TK, Langsford N, Ho YH, Smith RA and Lam AK: Regulation of microRNA-1288 in colorectal cancer: Altered expression and its clinicopathological significance. Mol Carcinog 53 (Suppl 1): E36-E44, 2014.

10. Gopalan V, Islam F, Pillai S, Tang JC, Tong DK, Law S, Chan KW and Lam AK: Overexpression of microRNA-1288 in oesophageal squamous cell carcinoma. Exp Cell Res 348: 146-154, 2016.

11. Livak KJ and Schmittgen TD: Analysis of relative gene expression data using real-time quantitative PCR and the 2(-Delta Delta C(T)) method. Methods 25: 402-408, 2001.

12. Zheng F, Zhang J, Luo S, Yi J, Wang P, Zheng Q and Wen Y: miR-143 is associated with proliferation and apoptosis involving ERK5 in HeLa cells. Oncol Lett 12: 3021-3027, 2016.

13. Shen L, Liu L, Ge L, Xie L, Liu S, Sang L, Zhan T and Li H: miR-448 downregulates MPPED2 to promote cancer proliferation and inhibit apoptosis in oral squamous cell carcinoma. Exp Ther Med 12: 2747-2752, 2016.

14. Tao WY, Wang CY, Sun YH, Su YH, Pang D and Zhang GQ: MicroRNA-34c suppresses breast cancer migration and invasion by targeting GIT1. J Cancer 7: 1653-1662, 2016.

15. Song L, Yang J, Duan P, Xu J, Luo X, Luo F, Zhang Z, Hou T, Liu B and Zhou Q: MicroRNA-24 inhibits osteosarcoma cell proliferation both in vitro and in vivo by targeting LPAAT $\beta$. Arch Biochem Biophys 535: 128-135, 2013.

16. Pan Y, Robertson G, Pedersen L, Lim E, Hernandez-Herrera A, Rowat AC, Patil SL, Chan CK, Wen Y, Zhang X, et al: miR-509-3p is clinically significant and strongly attenuates cellular migration and multi-cellular spheroids in ovarian cancer. Oncotarget 7: 25930-25948, 2016. 
17. Liu J, Xu J, Li H, Sun C, Yu L, Li Y, Shi C, Zhou X, Bian X, Ping Y, et al: miR-146b-5p functions as a tumor suppressor by targeting TRAF6 and predicts the prognosis of human gliomas. Oncotarget 6: 29129-29142, 2015.

18. Xue J, Zhou A, Wu Y, Morris SA, Lin K, Amin S, Verhaak R, Fuller G, Xie K, Heimberger AB and Huang S: miR-182-5p induced by STAT3 activation promotes glioma tumorigenesis. Cancer Res 76: 4293-4304, 2016.

19. Guo E, Wang Z and Wang S: MiR-200c and miR-141 inhibit ZEB1 synergistically and suppress glioma cell growth and migration. Eur Rev Med Pharmacol Sci 20: 3385-3391, 2016.

20. Gopalan V, Islam F, Pillai S, Tang JC, Tong DK, Law S, Chan KW and Lam AK: Overexpression of microRNA-1288 in oesophageal squamous cell carcinoma. Exp Cell Res 2016.

21. Bignell GR, Warren W, Seal S, Takahashi M, Rapley E, Barfoot R, Green $\mathrm{H}$, Brown C, Biggs PJ, Lakhani SR, et al: Identification of the familial cylindromatosis tumour-suppressor gene. Nat Genet 25: 160-165, 2000
22. Massoumi R: CYLD: A deubiquitination enzyme with multiple roles in cancer. Future Oncol 7: 285-297, 2011.

23. Hayashi M, Jono H, Shinriki S, Nakamura T, Guo J, Sueta A, Tomiguchi M, Fujiwara S, Yamamoto-Ibusuki M, Murakami K, et al: Clinical significance of CYLD downregulation in breast cancer. Breast Cancer Res Treat 143: 447-457, 2014.

24. Zhang L, Ding Y, Yuan Z, Liu J, Sun J, Lei F, Wu S, Li S and Zhang D: MicroRNA-500 sustains nuclear factor- $x$ B activation and induces gastric cancer cell proliferation and resistance to apoptosis. Oncotarget 6: 2483-2495, 2015.

25. Ge WL, Xu JF and Hu J: Regulation of oral squamous cell carcinoma proliferation through crosstalk between SMAD7 and CYLD. Cell Physiol Biochem 38: 1209-1217, 2016. 